\title{
Palliativpflege in Pflegeheimen in New York State
}

\section{Bei vielen Pflegeheimbewohnern, die unter einer akuten lebensbedrohlichen oder chronischen Erkrankung leiden, kann die Symptomatik mit Hilfe von Palliativpflege reduziert und die Lebensqualität erhöht werden. Doch die Einführung von entspre- chenden Programmen, insbesondere die schmerztherapeutische Behandlung, stellt die Mitarbeiter von Pflegeheimen oft vor große Herausforderungen.}

» Die Palliativpflege konzentriert sich generell auf eine Pflege, deren Fokus auf der Linderung von Symptomen, Schmerzen sowie Stress liegt. Ziel ist es, die Lebensqualität des Patienten solange wie möglich zu erhalten oder wieder herzustellen. Palliativpflege verfolgt einen ganzheitlichen Ansatz und wird von einem interdisziplinären Team aus Ärzten, Pflegepersonal, Psychologen, Seelsorgern, Sozialarbeitern, ehrenamtlichen Helfer und weiteren Berufen im Gesundheitswesen begleitet. Studien ergaben, dass Palliativpflege in Pflegeheimen mit einem besseren Schmerzmanagement und weniger Hospitalisierung in den letzten Monaten des Lebens im Zusammenhang steht.

In den USA existiert seit einigen Jahren eine Projektgruppe, die sich mit der Implementierung von bestehenden und neuen Strukturen der Palliativpflege beschäftigt, um diese Programme in den Pflegeheimen zu fördern und $\mathrm{zu}$ begleiten. Seitdem fand eine dynamische Entwicklung statt. Die hier vorgestellte Studie von Lester et al. (2017) untersuchte die aktuellen Entwicklungen in Pflegeheimen im Staat New York.

\section{Probleme bei der Finanzierung}

Die Studie wurde mittels Onlinefragebogen durchgeführt und von 149 Teilnehmern, die aus 61 verschiedenen Pflegeheimen kamen, ausgefüllt. An der Befragung haben sich Ärzte, Pflegepersonal, Sozialarbeiter und weitere Berufsgruppen beteiligt. Dabei stellte sich heraus, dass weniger Hospiz- als Palliativpflege durchgeführt wird. Die Ärzte bildeten die größte Berufsgruppe im Team, wobei die Trauerbegleiter den geringsten Teil ausmachten. Allgemein bestand in der Gruppe der Befragten Einigkeit über die positiven Auswirkungen von Palliativpflege. Der Frage bezüglich der Wahrscheinlichkeit, dass Bewohner aufgrund von Palliativpflege im Pflegeheim anstatt im Krankenhaus sterben, stimmten $42 \%$ der Pflegekräfte und 88,9\% der Ärzte und der medizinischen Leitung zu. Für die Implementierung von Palliativpflege wurden von den Befragten zwei Barrieren benannt: zum einem die Finanzierung solcher Programme in Pflegeheimen und zum anderen ausgebildetes Personal speziell für die Palliativpflege zu finden.

\section{Kommentar}

Grundsätzlich sollte man die Ergebnisse der Studie nicht verallgemeinern, da das amerikanische Gesundheitswesen anders strukturiert ist als in Deutschland. Unterschiede lassen sich beispielsweise in der Struktur der Pflegeheime, der Personalausstattung sowie in den Kosten für die Pflege ausmachen. Die Autoren der Studie weisen jedoch auf die limitierte Stichprobengröße hin und äußern Bedenken, weil keine einheitliche Nutzung von Palliativpflegeprogrammen stattgefunden hat. Die Studie beinhaltet als Anhang die komplette Darstellung der Fragen und verschafft dadurch einen guten Überblick. Der Fragebogen bestand zum einen aus geschlossenen Fragen und zum anderen aus einer offenen Frage, um die Empfindungen und Reaktionen der Befragten einzuholen. Über eine Skala von 1 bis 5 (,stimme voll ganz zu“ bis zu „stimme überhaupt nicht zu“) wurden Einstellungen über Palliativpflege in Pflegeheimen erfasst. 